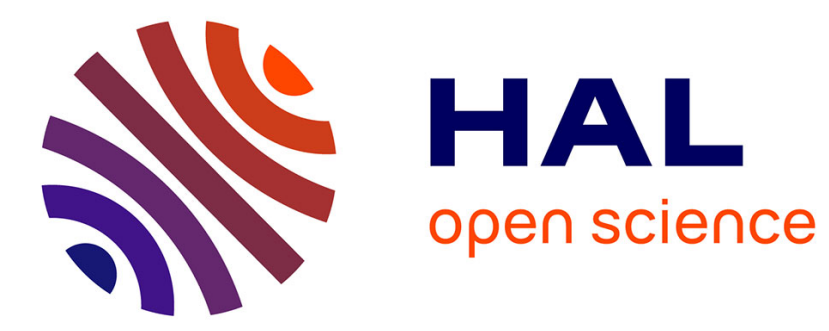

\title{
Conformal Geometry for Viewpoint Change Representation
}

\author{
Ghina El Mir, Christophe Saint-Jean, Michel Berthier
}

\section{To cite this version:}

Ghina El Mir, Christophe Saint-Jean, Michel Berthier. Conformal Geometry for Viewpoint Change Representation. Advances in Applied Clifford Algebras, 2014, 24 (2), pp.443-463. hal-00831744

\section{HAL Id: hal-00831744 https://hal.science/hal-00831744}

Submitted on 10 Jun 2013

HAL is a multi-disciplinary open access archive for the deposit and dissemination of scientific research documents, whether they are published or not. The documents may come from teaching and research institutions in France or abroad, or from public or private research centers.
L'archive ouverte pluridisciplinaire HAL, est destinée au dépôt et à la diffusion de documents scientifiques de niveau recherche, publiés ou non, émanant des établissements d'enseignement et de recherche français ou étrangers, des laboratoires publics ou privés. 


\title{
Conformal Geometry for Viewpoint Change Representation
}

\author{
Ghina El Mir, Christophe Saint-Jean and Michel Berthier
}

\begin{abstract}
We propose in this paper a new model for image representation based on the conformal geometry and its powerfulness to encode perspective distortions through bases of the Minkowski space $\mathbb{R}^{1,1}$. This approach allows us to describe an image as a scalar valued function defined on a horosphere corresponding to an embedding of the Euclidean plane into $\mathbb{R}^{3,1}$ encoding the latitude angle and the rotation parameter of the camera. This is obtained through a generalization of the conformal model such that it includes representations of perspective planes. In this setting, we describe every viewpoint change as a mapping between two horospheres of the space $\mathbb{R}^{3,1}$, each one of these encoding a perspective plane.
\end{abstract}

Computer Vision, Pinhole Camera, Conformal Geometry, Image Representation, Perspective Distortions, Geometric Algebra, Perspective Plane.

\section{Introduction}

In computer vision, the search for viewpoint invariant detectors and descriptors is still an important problem mainly for strong differences in the viewpoints. An interesting solution relies on representing all the viewpoint changes by one useful topological group:

- There are well defined viewpoint invariants in the case where a generic group acts on the set of images, see [11].

- Some authors already used this setting and found invariants to the actions of some particular groups such as the group of planar motions, see [1].

In order to achieve this goal, a fundamental step needs to be accomplished first: the construction of a powerful image representation leading to an efficient modeling of every viewpoint change. This requires a unified mathematical framework allowing useful computations of well defined viewpoint invariants (see for instance [11]). 
The most common image representation is the pinhole camera model which relies on the perspective projection of points in the 3-D space onto the image plane. This is represented even by $3 \times 4$ matrices within matrix algebra, or by the meet of a bivector with a trivector within projective geometric algebra. However, these two representations are useful for a 3-D reconstruction of a scene not for a viewpoint invariance modeling.

We propose in this work to make use of more powerful mathematical tool, the conformal geometric algebra, and to describe an image and a single viewpoint change within the conformal model. For this, we introduce a generalization of the conformal model of the Euclidean plane preserving its properties and extending it to encode the perspective planes that will be defined in this paper. This leads us to describe a new conformal image representation corresponding to an embedding of $\mathbb{R}^{2}$ into the space $\mathbb{R}^{3,1}$ encoding the latitude angle of the camera (which is the parameter that produces the main deformation of the planar object), as well as the parameter of the rotation of the camera about its optical axis. Thus an image is defined as a mapping on a horosphere with a given such embedding. Within this setting, the 8 parameters of the homography of the real projective plane that describes a viewpoint change will be explicitly modeled through a mapping between two horospheres. This can particularly be applied to encode the pinhole model of acquisition of an image by modeling the viewpoint change between this image and the reflectance of the planar object.

We will discuss in a future work how to consider these representations in order to construct a subgroup of the linear conformal group of $\mathbb{R}^{3,1}$ that models all possible viewpoint changes. This will allow us to introduce the action of this subgroup on the set of conformal image representations in order to define viewpoint change invariants.

The rest of this paper is organized as follows. Section 2 gives some definitions and background on projective geometry, geometric algebra and the conformal space. The aim in section 3 is to represent the camera extrinsic parameters as well as every viewpoint change in projective geometry. In section 4 we deal with the conformal model and define an extension to this one enabling us to represent perspective planes. This is used to construct an image representation within conformal geometry leading to conformal viewpoint change modeling. In the end of this section, we apply the previous viewpoint change representation to describe the pinhole camera in conformal geometry.

\section{Preliminaries and basic definitions}

Projective geometry has been used since a long time ago in computer vision (see [2], [8]). It is one of the main geometries that are used to represent the pinhole camera model. In this setting, two images of a planar object from different viewpoints are related by a homography of the real projective plane $\mathbb{P}_{2}(\mathbb{R})$. At first sight, this may look useful for the search of viewpoint invariants, since the cross ratio of four collinear points is the scalar valued function 
that is preserved under homographies of $\mathbb{P}_{2}(\mathbb{R})$ (see $[8]$ ). However, this function is not efficient as an invariant image descriptor for several reasons: it depends on four points, which must belong to the same line and must be sorted. This suggests to restrict the search to some special kinds of homographies of $\mathbb{P}_{2}(\mathbb{R})$, such as affine transformations (see [12]) and dilations (see [7]). Nevertheless, since these transformations can't approximate or represent generic homographies of $\mathbb{P}_{2}(\mathbb{R})$, the ensuing algorithms fail to be robust to strong viewpoint changes.

\subsection{Projective geometry within geometric algebra}

We first review some definitions and background. For further details about geometric algebra, the reader is referred to [3], [4] and [5].

The geometric algebra associated with a real vector space $E$, of finite dimension $n$, equipped with a quadratic form $Q$, is an associative algebra that contains $\mathbb{R}$ as a sub-algebra and $E$ as a subspace, and in which:

$$
v^{2}=Q(v)
$$

for all $v$ in $E$. If $Q$ is a quadratic form of signature $(p, q)$ on $\mathbb{R}^{n}$, where $p+q=n$, we denote by $\mathbb{R}^{p, q}$ the corresponding vector space and by $\mathbb{R}_{p, q}$ its geometric algebra. Particularly, the Euclidean space is denoted by $\mathbb{R}^{n}$ and its geometric algebra by $\mathbb{R}_{n}$. The outer product of two vectors $u$ and $v$, is the element of $\mathbb{R}_{p, q}$ defined by:

$$
u \wedge v:=u v-u \cdot v
$$

An isotropic blade $A$ (see [3]) is a blade that squares to zero: $A^{2}=0$.

Definition 2.1. Let $A_{r}$ be an $r$-blade that is not isotropic, and $X$ be a vector in $\mathbb{R}^{p, q}$. The projection $P_{A_{r}}(X)$ of $X$ onto the sub-space of $A_{r}$ is defined by

$$
P_{A_{r}}(X)=\left(X \cdot A_{r}\right) A_{r}^{-1}
$$

and the orthogonal rejection $P_{A_{r}}^{\perp}(X)$ of $X$ from the sub-space of $A_{r}$ is

$$
P_{A_{r}}^{\perp}(X)=\left(X \wedge A_{r}\right) A_{r}^{-1} .
$$

A $k$-versor is a multivector that can be factorized into the geometric product of $k$ unit vectors. The set of all versors is a group under the geometric product and more precisely a double covering of the orthogonal group of $\mathbb{R}^{p, q}$. A spinor is an even versor, i.e a versor with an even $k$.

Definition 2.2. Let $M$ and $N$ be two blades such that grade $(M)+$ grade $(N) \geq n$. The dual $\widetilde{M}$ of $M$ is defined by:

$$
\widetilde{M}=M I_{n}^{-1},
$$

where $I_{n}$ is the unit pseudoscalar (see [3]). Furthermore, if $M$ and $N$ satisfy:

$$
\widehat{M}+\widehat{N}=\mathbb{R}^{p, q},
$$

where $\widehat{M}$ and $\widehat{N}$ denote the two sub-spaces of $\mathbb{R}^{p, q}$ represented by $M$ and $N$ respectively (see [3]), then the meet $M \vee N$ of $M$ and $N$ is given by:

$$
M \vee N=(\widetilde{M} \wedge \widetilde{N}) I_{n}
$$


In his Ph.D thesis [9], Perwass embeds the Euclidean plane $\mathbb{R}^{2}$ into the real projective plane of the same dimension. An extra dimension enables to homogenize the Euclidean plane through the operation:

$$
\begin{aligned}
\mathbb{R}^{2} & \longrightarrow \mathbb{R}^{3} \\
x & \longmapsto X=x+e_{3}
\end{aligned}
$$

In other words, the affine plane of $\mathbb{R}^{3}$ given by the set

$$
\left\{X \in \mathbb{R}^{3} ; X=x+e_{3} \text { and } x \in \mathbb{R}^{2}\right\},
$$

constitutes the homogeneous representation of the Euclidean plane. Then, the space $\mathbb{R}^{3}$ is embedded into its Euclidean geometric algebra $\mathbb{R}_{3}$ in order to do some representations of geometric objects and operations.

Since the meet represents the intersection operation, the pinhole camera model is interpreted by an algebraic identity instead of using matrices. That is, the image of a homogeneous point $X$ is the meet of a bivector with a trivector. The bivector represents the line through $X$ and the optical center of the camera, while the trivector represents the image plane. Nevertheless, this representation is used to make a $3-\mathrm{D}$ reconstruction of a scene not for a group-modeling of the viewpoint changes.

\subsection{The conformal geometry}

The conformal model realizes an embedding of the Euclidean plane $\mathbb{R}^{2}$ in a 4-dimensional vector space (for details see [10] and [6]). This embedding is obtained as follows:

$$
\widehat{\mathbb{R}^{2}} \underbrace{\equiv}_{P^{-1}} S^{2} \underbrace{\hookrightarrow}_{\text {hom }} \mathbb{R}^{3,1}
$$

where $\widehat{\mathbb{R}^{2}}$ denotes the Riemann sphere, $P$ represents the stereographic projection and hom is the homogenization (see [10] pages 54 to 58). Let the two isotropic vectors of $\mathbb{R}^{1,1}$ :

$$
\begin{aligned}
e_{\infty, \alpha} & =(\alpha, \alpha) \\
e_{0, \frac{1}{\alpha}} & =\frac{1}{2}\left(-\frac{1}{\alpha}, \frac{1}{\alpha}\right),
\end{aligned}
$$

where $e_{\infty, \alpha} \cdot e_{0, \frac{1}{\alpha}}=-1$, for all $\alpha \neq 0$. Then the set $\left\{e_{+, \alpha}, e_{-, \alpha}\right\}$ given by:

$$
\begin{aligned}
e_{\infty, \alpha} & =e_{+, \alpha}+e_{-, \alpha} \\
e_{0, \frac{1}{\alpha}} & =\left(e_{-, \alpha}-e_{+, \alpha}\right) / 2
\end{aligned}
$$

is an orthonormal basis of $\mathbb{R}^{1,1}$. In fact, these two bases satisfy the following: $\left(e_{0, \frac{1}{\alpha}}\right)^{2}=0=\left(e_{\infty, \alpha}\right)^{2}$ and $e_{\infty, \alpha} \cdot e_{0, \frac{1}{\alpha}}=-1$ if and only if $\left\{e_{+, \alpha}, e_{-, \alpha}\right\}$ is an orthonormal basis of $\mathbb{R}^{1,1}$. The previous isotropic vectors are of great importance in the conformal model, as well as for the image representation described below. They will be used to encode the extrinsic parameter of the camera which is difficult to deal with in the projective space : the latitude angle, that is the parameter corresponding to the perspective distortion of 
an image (see Sec. 4). The space $\mathbb{R}^{3,1}$ can be decomposed into the following direct sum:

$$
\mathbb{R}^{3,1}=\mathbb{R}^{2} \oplus \mathbb{R}^{1,1}
$$

called a conformal split, where $\left\{e_{1}, e_{2}\right\}$ denotes an orthonormal basis of $\mathbb{R}^{2}$. This decomposition is uniquely determined by the pseudoscalar

$$
E=e_{\infty, \alpha} \wedge e_{0, \frac{1}{\alpha}}=e_{+, \alpha} \wedge e_{-, \alpha}
$$

which is independent of the scalar $\alpha$ (see equation (11)): let the vector

$$
X_{\alpha}=x_{1} e_{1}+x_{2} e_{2}+x_{\infty} e_{\infty, \alpha}+x_{0} e_{0, \frac{1}{\alpha}}
$$

in the space $\mathbb{R}^{3,1}$, then

$$
X_{\alpha}=P_{E}^{\perp}\left(X_{\alpha}\right)+P_{E}\left(X_{\alpha}\right)
$$

where

$$
P_{E}^{\perp}\left(X_{\alpha}\right)=\left(X_{\alpha} \wedge E\right) E=x_{1} e_{1}+x_{2} e_{2}
$$

is the rejection of $X_{\alpha}$ from the $E$-plane and

$$
P_{E}\left(X_{\alpha}\right)=\left(X_{\alpha} \cdot E\right) E=x_{\infty} e_{\infty, \alpha}+x_{0} e_{0, \frac{1}{\alpha}}
$$

is the projection of $X_{\alpha}$ onto the $E$-plane.

Through the previous embedding of $\mathbb{R}^{2}$ in the space $\mathbb{R}_{3,1}$ (equation (10)), the conformal representation of the Euclidean space, called horosphere, is defined as follows:

Definition 2.3. The horosphere denoted by $H_{\alpha}$ is the conformal model of $\mathbb{R}^{2}$ associated with the basis $\left\{e_{\infty, \alpha}, e_{0, \frac{1}{\alpha}}\right\}$ and is the following set of normalized isotropic vectors of $\mathbb{R}^{3,1}$ :

$$
H_{\alpha}=\left\{X_{\alpha} \in \mathbb{R}^{3,1} ; X_{\alpha}^{2}=0 \text { and } X_{\alpha} \cdot e_{\infty, \alpha}=-1\right\} .
$$

It's given by $H_{\alpha}=\varphi_{\alpha}\left(\mathbb{R}^{2}\right)$ where $\varphi_{\alpha}$ is the bijection $\varphi_{\alpha}: \mathbb{R}^{2} \longrightarrow H_{\alpha}$ that sends $x_{1} e_{1}+x_{2} e_{2}$ to

$$
X_{\alpha}=\varphi_{\alpha}\left(x_{1} e_{1}+x_{2} e_{2}\right)=x_{1} e_{1}+x_{2} e_{2}+\frac{1}{2}\left(x_{1}^{2}+x_{2}^{2}\right) e_{\infty, \alpha}+e_{0, \frac{1}{\alpha}} .
$$

One of the most important results of this model is that every Möbius transform of the Euclidean plane is linearized by the embedding (see [6] for instance). First, we remind the definition of a Möbius transformation of $\mathbb{R}^{2}$.

Definition 2.4. Let $A, B, C$ and $D$ be versors in $\mathbb{R}_{2}$ satisfying:

$$
\begin{gathered}
A B^{\dagger}, B D^{\dagger}, C D^{\dagger}, A C^{\dagger} \in \mathbb{R}^{2} \\
A D^{\dagger}-B C^{\dagger} \neq 0,
\end{gathered}
$$

where $\dagger$ denotes the reversion of the Clifford algebra. Then the Möbius transformation $f$ of $\mathbb{R}^{2}$ represented by the matrix

$$
[G]=\left(\begin{array}{cc}
A & B \\
C & D
\end{array}\right)
$$

is given by:

$$
f(x)=(A x+B)(C x+D)^{-1},
$$


for all $x \in \mathbb{R}^{2}$. The matrix $[G]$ is known as Vahlen matrix.

We have the following linearization result. The reader is referred to see [6] for all the details about the representations of the basic particular Möbius transformations.

Theorem 2.1. Let $f$ be a Möbius transformation of $\mathbb{R}^{2}$ given by the Vahlen matrix $[G]$. The versor

$$
G_{\alpha}=e_{\infty, \alpha}\left(-e_{0, \frac{1}{\alpha}} A+B\right)-e_{0, \frac{1}{\alpha}}\left(C+e_{\infty, \alpha} D\right)
$$

satisfies for all $x \in \mathbb{R}^{2}$ :

$$
G_{\alpha}\left(x+\frac{1}{2} x^{2} e_{\infty, \alpha}+e_{0, \frac{1}{\alpha}}\right)\left(G_{\alpha}^{*}\right)^{-1}=\sigma_{f}(x)\left[f(x)+\frac{1}{2}[f(x)]^{2} e_{\infty, \alpha}+e_{0, \frac{1}{\alpha}}\right],
$$

where

$$
\sigma_{f}(x)=(C x+D)\left(C^{*} x+D^{*}\right)^{\dagger}
$$

is a scalar valued function and $*$ denotes the main involution of the Clifford algebra.

Proposition 2.1 (Horospheres intersection). Let $H_{\alpha_{1}}$ and $H_{\alpha_{2}}$ be two horospheres given by:

$$
\begin{aligned}
& H_{\alpha_{1}}=\left\{X_{\alpha_{1}} \in \mathbb{R}^{3,1}, \text { s.t. } X_{\alpha_{1}}=x+\frac{1}{2} x^{2} e_{\infty, \alpha_{1}}+e_{0, \frac{1}{\alpha_{1}}} ; x \in \mathbb{R}^{2}\right\} \\
& H_{\alpha_{2}}=\left\{X_{\alpha_{2}} \in \mathbb{R}^{3,1} \text {, s.t. } X_{\alpha_{2}}=y+\frac{1}{2} y^{2} e_{\infty, \alpha_{2}}+e_{0, \frac{1}{\alpha_{2}}} ; y \in \mathbb{R}^{2}\right\}
\end{aligned}
$$

where

$$
e_{\infty, \alpha_{1}}=\left(\alpha_{1}, \alpha_{1}\right), e_{\infty, \alpha_{2}}=\left(\alpha_{2}, \alpha_{2}\right)
$$

and

$$
e_{0, \frac{1}{\alpha_{1}}}=\frac{1}{2}\left(-\frac{1}{\alpha_{1}}, \frac{1}{\alpha_{1}}\right), e_{0, \frac{1}{\alpha_{2}}}=\frac{1}{2}\left(-\frac{1}{\alpha_{2}}, \frac{1}{\alpha_{2}}\right)
$$

Then, the previous horospheres satisfy:

$$
H_{\alpha_{1}} \cap H_{\alpha_{2}} \neq \phi \quad \Longleftrightarrow H_{\alpha_{1}} \equiv H_{\alpha_{2}} \Longleftrightarrow \alpha_{1}=\alpha_{2} .
$$

Proof. Suppose that there exist $x$ and $y$ in $\mathbb{R}^{2}$ such that

$$
x+\frac{1}{2} x^{2} e_{\infty, \alpha_{1}}+e_{0, \frac{1}{\alpha_{1}}}=y+\frac{1}{2} y^{2} e_{\infty, \alpha_{2}}+e_{0, \frac{1}{\alpha_{2}}} .
$$

Since $\mathbb{R}^{3,1}=\mathbb{R}^{2} \oplus \mathbb{R}^{1,1}$, the last equation is equivalent to:

$$
\begin{gathered}
x=y \text { and } \frac{1}{2} x^{2} e_{\infty, \alpha_{1}}+e_{0, \frac{1}{\alpha_{1}}}=\frac{1}{2} y^{2} e_{\infty, \alpha_{2}}+e_{0, \frac{1}{\alpha_{2}}} \\
\Leftrightarrow x=y \text { and }\left\{\begin{array}{l}
x^{2} \alpha_{1}-\frac{1}{\alpha_{1}}=x^{2} \alpha_{2}-\frac{1}{\alpha_{2}} \\
x^{2} \alpha_{1}+\frac{1}{\alpha_{1}}=x^{2} \alpha_{2}+\frac{1}{\alpha_{2}}
\end{array}\right. \\
\Leftrightarrow x=y \text { and } \alpha_{1}=\alpha_{2}
\end{gathered}
$$

Therefore, two different horospheres, i.e. corresponding to different isotropic bases of $\mathbb{R}^{1,1}$ (equation (11)), do not intersect. 


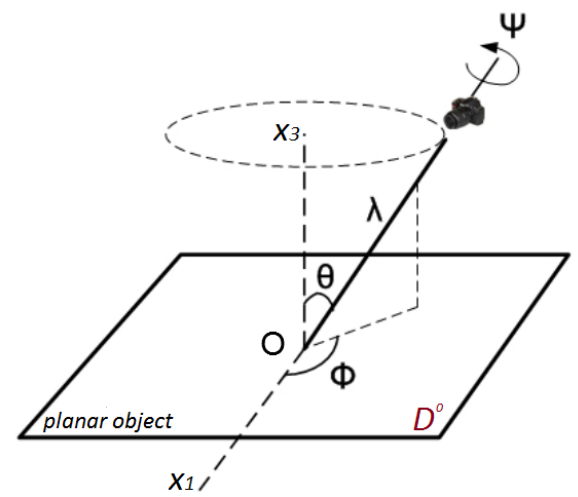

FiguRE 1. Viewpoint degrees of freedom for $t_{1}=t_{2}=0$ (from [12]).

\section{Projective representations}

Suppose that a planar object is embedded into the subspace $\mathbb{R}^{2} \times\{0\}$ of the three-dimensional space $\mathbb{R}^{3}$ equipped with a Cartesian coordinate system $\left(O, x_{1}, x_{2}, x_{3}\right)$ and an orthonormal basis $\left\{e_{1}, e_{2}, e_{3}\right\}$. We assume that the image $D^{0} \subset \mathbb{R}^{2} \times\{0\}$ of this embedding contains the origin $O$. The frame $\left(O, e_{1}, e_{2}, e_{3}\right)$ is called object frame.

\subsection{The camera extrinsic parameters}

We suppose that the camera is fully calibrated such that its intrinsic parameters are given by the matrix $K=I d$. In [12], the authors define the camera extrinsic parameters by making use of a spherical coordinate system for the space $\mathbb{R}^{3}$. More precisely, we have the following definition.

Definition 3.1. The camera extrinsic parameters are given by the 6-tuple $\left(\theta, \phi, \psi, \lambda, t_{1}, t_{2}\right)$ (see figure 1$)$ where:

1. The latitude angle $\theta$, which is the non-signed angle between the $z$-axis and the optical axis of the camera. We assume that $0 \leq \theta<\pi / 2$ radians.

2. The longitude angle $\phi($ for $\theta \neq 0)$, which is the signed angle between the $x_{1}$-axis and the orthogonal projection of the optical axis onto the plane $\left(O, x_{1}, x_{2}\right)$. We suppose that $0 \leq \phi<2 \pi$ radians.

3. The rotation signed angle $\psi$ of the camera about its optical axis $(0 \leq$ $\psi<2 \pi)$.

4. The zoom parameter $\lambda$ which is the distance between the position of the optical center $C$ of the camera in the space and the point $O^{\prime}$ of intersection of the optical axis with the object plane $(\lambda>0)$.

5 . The two parameters $t_{1}$ and $t_{2}$ of the planar translation that the camera can undergo parallel to the plane $\left(O, x_{1}, x_{2}\right)$.

Remark 3.1. The zoom parameter measures how far is the camera from the object plane. The angles $\theta$ and $\phi$ represent the direction of the optical axis. 
If the translation parameters are null, i.e. the camera axis passes through the origin $O$, then $(\lambda, \theta, \phi)$ are the spherical coordinates of the optical center and $(\lambda \sin \phi \sin \theta, \lambda \cos \phi \sin \theta, \lambda \sin \phi)$ are its Cartesian coordinates in the object frame. For $\theta=0$ the image is called a frontal image and corresponds to a frontal viewpoint. As for every $\theta \neq 0$ the longitude $\phi$ can take any arbitrary value in $[0,2 \pi[$, we suppose that this is also satisfied for the frontal case.

Indeed, as described in [2], the camera extrinsic parameters are the parameters encoding the $3-\mathrm{D}$ rotation and translation that relate the object frame to the camera frame in the 3 -D space. In fact, the camera frame denoted by $\left(C, e_{1}^{\prime}, e_{2}^{\prime}, e_{3}^{\prime}\right)$ is constructed through the object frame $\left(O, e_{1}, e_{2}, e_{3}\right)$ as follows (see figure 2):

First we apply to the object frame a translation $T_{e_{3}}$ vertically towards $C$ of vector $e_{3}$. This is followed by a rotation $R_{e_{3}}^{\psi}$ about $e_{3}$ of angle $\psi$ in order to allow the rotation of the camera about its optical axis. Next, the previous definitions of the latitude and longitude angles enables us to describe their actions by a two rotations, the first one $R_{e_{2}}^{\theta}$ about $e_{2}$ and of angle $\theta$ and the second one $R_{e_{3}}^{\phi}$ about $e_{3}$ and of angle $\phi$. Then the zoom parameter induces a dilation $D_{\lambda}$ of ratio $\lambda$. These transformations of the space map the origin $O$ onto the vector $\overrightarrow{u^{\prime}}$ (see figure 2 ) given by:

$$
\overrightarrow{u^{\prime}}=D_{\lambda} R_{e_{3}}^{\phi} R_{e_{2}}^{\theta} R_{e_{3}}^{\psi} T_{e_{3}}(O)=\lambda \sin \phi \sin \theta e_{1}+\lambda \cos \phi \sin \theta e_{2}+\lambda \sin \phi e_{3} .
$$

Finally, the coordinates of $O^{\prime}$ which represent the planar translation of the camera are taken into account by applying a translation $T_{t_{1}, t_{2}}$ by the vector $\left(t_{1}, t_{2}, 0\right)$. Thus, the 3 -D transformation that expresses the camera frame according to the object frame is decomposed as follows:

$$
l=T_{t_{1}, t_{2}} D_{\lambda} R_{e_{3}}^{\phi} R_{e_{2}}^{\theta} R_{e_{3}}^{\psi} T_{e_{3}} .
$$

The camera frame is then defined by $\left(C, e_{1}^{\prime}, e_{2}^{\prime}, e_{3}^{\prime}\right)$ associated with the Cartesian coordinate system $\left(C, x_{1}^{\prime}, x_{2}^{\prime}, x_{3}^{\prime}\right)$, where

$$
\begin{aligned}
\overrightarrow{O C} & =l(O) \\
e_{1}^{\prime} & =R_{e_{3}}^{\phi} R_{e_{2}}^{\theta} R_{e_{3}}^{\psi}\left(e_{1}\right) \\
e_{2}^{\prime} & =R_{e_{3}}^{\phi} R_{e_{2}}^{\theta} R_{e_{3}}^{\psi}\left(e_{2}\right) \\
e_{3}^{\prime} & =R_{e_{3}}^{\phi} R_{e_{2}}^{\theta} R_{e_{3}}^{\psi}\left(e_{3}\right) .
\end{aligned}
$$

The image plane equation in the camera frame is given by: $x_{3}^{\prime}=-1$.

Hereafter, let $x=x_{1} e_{1}+x_{2} e_{2}=O \vec{M}$ be a vector encoding the coordinates of a point $M$ in the object frame. This point can be expressed in the camera frame by

$$
\begin{aligned}
x^{\prime} & =l^{-1}(x) \\
& =T_{-e_{3}} R_{e_{3}}^{-\psi} R_{e_{2}}^{-\theta} R_{e_{3}}^{-\phi} D_{\lambda^{-1}} T_{-t_{1},-t_{2}}(x) .
\end{aligned}
$$

That is, if $x^{\prime}=O \vec{M}^{\prime}=x_{1}^{\prime} e_{1}+x_{2}^{\prime} e_{2}+x_{3}^{\prime} e_{3}$ then $C \vec{M}=x_{1}^{\prime} e_{1}^{\prime}+x_{2}^{\prime} e_{2}^{\prime}+x_{3}^{\prime} e_{3}^{\prime}$ i.e. the coordinates of $M^{\prime}$ in the object frame are equal to those of $M$ expressed in the camera frame. Thus, the projection of $M$ onto the image plane $x_{3}^{\prime}=-1$ 


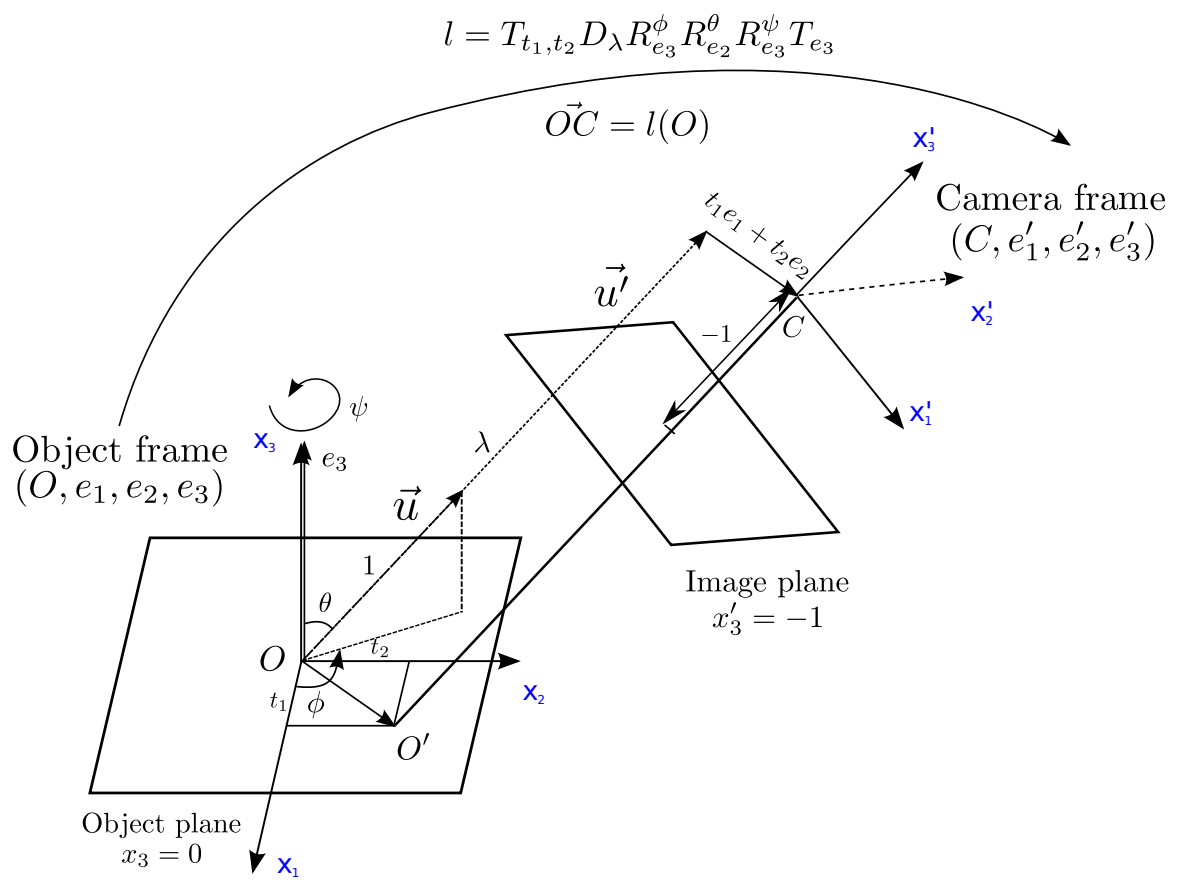

Figure 2. Construction of the camera frame $\left(C, e^{\prime}{ }_{1}, e_{2}^{\prime}, e_{3}^{\prime}\right)$ through the object frame $\left(O, e_{1}, e_{2}, e_{3}\right)$. The 3 -D transformation describing this construction is denoted by $l$ (see equations (37) and (38)). The parameters of $l$ are the camera six extrinsic parameters.

is identified to the projection of $M^{\prime}$ onto the plane $x_{3}=-1$. Let then $\Pi$ denote the projection onto the plane $x_{3}=-1$ such that

$$
\Pi\left(x_{1} e_{1}+x_{2} e_{2}+x_{3} e_{3}\right)=\left\{\begin{array}{cc}
-\frac{x_{1}}{x_{3}} e_{1}-\frac{x_{2}}{x_{3}} e_{2}-e_{3} & \text { if } x_{3} \neq 0 \\
x_{1} e_{1}+x_{2} e_{2} & \text { if } x_{3}=0
\end{array},\right.
$$

then the 3-D Euclidean transformation that expresses the image coordinates in the camera frame is given by: $x_{c a m}=\Pi\left(x^{\prime}\right)$. Thanks to commutativity between some elements, this can be written as:

$$
x_{c a m}=R_{e_{3}}^{-\psi}\left(\Pi T_{-e_{3}} R_{e_{2}}^{-\theta}\right) R_{e_{3}}^{-\phi} D_{\lambda^{-1}} T_{-t_{1},-t_{2}}(x) .
$$

This leads to interpret the pinhole camera model in the 2-D homogeneous space by the following identity:

$$
I=k_{0}^{-1} I^{0}:=I^{0} \circ k_{0}^{-1},
$$

where

$$
k_{0}=R_{-\psi} h_{\theta} R_{-\phi} D_{\lambda^{-1}} T
$$

and 
1. $I^{0}: \mathbb{R}^{2} \rightarrow \mathbb{R}$ is the frontal image corresponding to the extrinsic parameters $(0,0,0,1,0,0)$, i.e. representing the object reflectance (see [11]),

2. $T$ is a planar translation,

3. $R_{\psi}$ is a planar rotation about the origin with a rotation angle $\psi$,

4. $D_{\lambda}$ is a dilation centered on the origin with ratio $\lambda$,

5. $h_{\theta}:=\Pi T_{-e_{3}} R_{e_{2}}^{-\theta}$ is called perspective distortion and is represented in the 2 -D homogeneous space by the $3 \times 3$ matrix $M_{\theta}$ given by:

$$
M_{\theta}=\left(\begin{array}{ccc}
\cos \theta & 0 & 0 \\
0 & 1 & 0 \\
-\sin \theta & 0 & 1
\end{array}\right) \text {. }
$$

Therefore, an image of a planar object is represented by any mapping

$$
I_{\psi, \theta}: R_{-\psi} h_{\theta}\left(\mathbb{R}^{2}\right) \longrightarrow \mathbb{R} .
$$

If the corresponding extrinsic parameters of the camera $\left(\theta, \phi, \psi, \lambda, t_{1}, t_{2}\right)$ are known, then one can find the object reflectance $I^{0}$ through equations (42) and (43).

Remark 3.2. $\quad$ 1. It's immediate that in the case of frontal viewpoint, the perspective distortion is $h_{0}=I d$.

2. The inverse of $M_{\theta}$ is given by:

$$
M_{\theta}^{-1}=\frac{1}{\cos \theta}\left(\begin{array}{ccc}
1 & 0 & 0 \\
0 & \cos \theta & 0 \\
\sin \theta & 0 & \cos \theta
\end{array}\right) .
$$

Since it's a homogeneous matrix, we can omit the factor $\frac{1}{\cos \theta}$. It's a matrix representing $h_{\theta}^{-1}$ in the $2-D$ homogeneous space.

\subsection{Viewpoint changes in the projective geometry}

Through the previous representation of the acquisition of an image via the projective pinhole camera model, the homography $k$ of $\mathbb{P}_{2}(\mathbb{R})$ that relates two different views of the planar object can be written as:

$$
k: R_{-\psi_{1}} h_{\theta_{1}}\left(\mathbb{R}^{2}\right) \subset \mathbb{P}_{2}(\mathbb{R}) \longrightarrow R_{-\psi_{2}} h_{\theta_{2}}\left(\mathbb{R}^{2}\right) \subset \mathbb{P}_{2}(\mathbb{R})
$$

such that

$$
\begin{aligned}
k & =R_{-\psi_{2}} h_{\theta_{2}} f\left(R_{-\psi_{1}} h_{\theta_{1}}\right)^{-1} \\
& =R_{-\psi_{2}} h_{\theta_{2}} f h_{\theta_{1}}^{-1} R_{\psi_{1}},
\end{aligned}
$$

where $f$ is an affine similarity (of the $2-\mathrm{D}$ homogeneous space as well).

Since $R_{-\psi_{2}}$ and $h_{\theta_{2}}$ (resp. $h_{\theta_{1}}^{-1}$ and $R_{\psi_{1}}$ ) do not commute weakly, the homography $k$ has 8 degrees of freedom because the similarity $f$ has a four parameters. Let $\Gamma$ denotes the set of homographies of $\mathbb{P}_{2}(\mathbb{R})$ given by equation (46). In the next section, we will represent every $k \in \Gamma$ by a conformal mapping through the conformal model. 
Note that frontal viewpoint changes are represented by affine similarities $k \in \Gamma\left(\right.$ for $\left.\theta_{1}=\theta_{2}=0\right)$.

\section{Conformal representations}

The aim here is to encode every $k \in \Gamma$ which models a viewpoint change by a mapping in the conformal geometry. It's immediate, through equation (25) that a similarity $f \in \Gamma$ is represented in the conformal model by a bijection from a horosphere $H_{\alpha}$ onto itself given by

$$
X_{\alpha}=\varphi_{\alpha}(x) \longmapsto \delta G_{\alpha} X_{\alpha} G_{\alpha}^{-1}=f(x)+\frac{1}{2}[f(x)]^{2} e_{\infty, \alpha}+e_{0, \frac{1}{\alpha}} \in H_{\alpha},
$$

where $\delta$ is the ratio of $f\left(\sigma_{f}(x)=\delta^{-1}, \forall x \in \mathbb{R}^{2}\right)$, for an arbitrary $\alpha \neq 0$. However, the restriction $h_{\theta \mid \mathbb{R}^{2}}$ of the perspective distortion is not a Möbius transformation on $\mathbb{R}^{2}$, therefore it can't be represented by a versor in the conformal model of $\mathbb{R}^{2}$. Our approach is to link the choice of the parameter $\alpha$ of $H_{\alpha}$ to the latitude angle $\theta$ so that $\varphi_{\alpha}$ of equation (20) would encode $h_{\theta \mid \mathbb{R}^{2}}$ and thus a generic $k \in \Gamma$ for $\theta_{1} \neq \theta_{2}$ can be modeled by a mapping between two different horospheres $H_{\alpha_{1}}$ and $H_{\alpha_{2}}$ encoding $h_{\theta_{1}}\left(\mathbb{R}^{2}\right)$ and $h_{\theta_{2}}\left(\mathbb{R}^{2}\right)$ respectively.

\subsection{Conformal model of the perspective plane: A generalization of the con- formal model of the Euclidean plane}

As described above, for every scalar $\alpha \neq 0$ the horosphere $H_{\alpha}=\varphi_{\alpha}\left(\mathbb{R}^{2}\right)$ realizes a conformal modelling of the Euclidean plane $\mathbb{R}^{2}$. The same vector $x \in \mathbb{R}^{2}$ can be 'seen' through diverse conformal representations: for all $\alpha \neq 0$ its homogeneous representation within the conformal model of $\mathbb{R}^{2}$ associated with the isotropic vectors $e_{\infty, \alpha}$ and $e_{0, \frac{1}{\alpha}}$ is the vector $X_{\alpha}=\varphi_{\alpha}(x)$ where $x=P_{E}^{\perp}\left(X_{\alpha}\right)$ (see equation $(20)$ ).

Our purpose in this subsection is to generalize the conformal model so that it represents not only the Euclidean plane but also $h_{\theta}\left(\mathbb{R}^{2}\right)$ for all $\theta \in] 0, \frac{\pi}{2}[$. This generalization satisfies the following properties:

1. $\alpha$ encodes the latitude angle $\theta$ i.e. $\alpha=\alpha_{\theta}$ where $\theta \mapsto \alpha_{\theta}$ is bijective,

2. the horosphere $H_{\alpha_{\theta}}=\varphi_{\alpha_{\theta}}\left(\mathbb{R}^{2}\right)$ models $h_{\theta}\left(\mathbb{R}^{2}\right)$ for all $\left.\theta \in\right] 0, \frac{\pi}{2}[$,

3 . this is reduced to the conformal model of $\mathbb{R}^{2}$ for only one specific $\alpha=\alpha_{0}$ i.e. the horosphere $\varphi_{\alpha_{0}}\left(\mathbb{R}^{2}\right)$ encodes $h_{0}\left(\mathbb{R}^{2}\right)=\mathbb{R}^{2}$,

4. for every $\alpha_{\theta}$, the representations of Möbius transformations of $\mathbb{R}^{2}$ by versors in $\mathbb{R}_{3,1}$ (equation $(25)$ ) are preserved. The versors depend on the value of $\alpha_{\theta}$.

This leads us to construct a modeling of a generic $k \in \Gamma$ by a mapping between two horospheres.

We call a perspective plane the image $h_{\theta}\left(\mathbb{R}^{2}\right)$ of the Euclidean plane by a perspective distortion $h_{\theta}$, for all $\theta \in\left[0, \frac{\pi}{2}[\right.$. It is strictly included in the real projective plane $\mathbb{P}_{2}(\mathbb{R})$. 
First let $\theta \in] 0, \frac{\pi}{2}\left[\right.$ and $x=x_{1} e_{1}+x_{2} e_{2} \in \mathbb{R}^{2}$. The image $h_{\theta}\left(x_{1} e_{1}+x_{2} e_{2}\right)$ of $x$ by $h_{\theta}$ is represented in the projective geometry by the 2-D homogeneous vector:

$$
\begin{aligned}
v_{\theta}\left(x_{1} e_{1}+x_{2} e_{2}\right) & =M_{\theta} \cdot\left(x_{1} e_{1}+x_{2} e_{2}+e_{3}\right) \\
& =x_{1} \cos \theta e_{1}+x_{2} e_{2}+\left(-x_{1} \sin \theta+1\right) e_{3} .
\end{aligned}
$$

The $e_{3}$-component of $v_{\theta}\left(x_{1} e_{1}+x_{2} e_{2}\right)$ is equal to zero if and only if $h_{\theta}\left(x_{1} e_{1}+\right.$ $\left.x_{2} e_{2}\right)$ is a point at infinity of $\mathbb{P}_{2}(\mathbb{R})$ : for all $x_{2} \in \mathbb{R}, h_{\theta}\left(1 / \sin \theta e_{1}+x_{2} e_{2}\right)$ is a point at infinity represented by

$$
v_{\theta}\left(1 / \sin \theta e_{1}+x_{2} e_{2}\right)=\cot \theta e_{1}+x_{2} e_{2},
$$

where $\cot$ encodes the cotangent function. Since $e_{\infty, \alpha}$ models all the points at infinity, it encodes $h_{\theta}\left(1 / \sin \theta e_{1}+x_{2} e_{2}\right)$ for all $x_{2} \in \mathbb{R}$. We set then through the expression of the vector $v_{\theta}\left(1 / \sin \theta e_{1}+x_{2} e_{2}\right)$

$$
\begin{aligned}
e_{\infty, \alpha_{\theta}}=\left(\alpha_{\theta}, \alpha_{\theta}\right) & =(\cot \theta, \cot \theta) \\
e_{0, \frac{1}{\alpha_{\theta}}}=\frac{1}{2}\left(-\frac{1}{\alpha_{\theta}}, \frac{1}{\alpha_{\theta}}\right) & =\frac{1}{2}(-\tan \theta, \tan \theta),
\end{aligned}
$$

and the orthonormal basis $\left\{e_{+, \alpha_{\theta}}, e_{-, \alpha_{\theta}}\right\}$ is given by:

$$
\left\{\begin{array}{c}
e_{0, \frac{1}{\alpha_{\theta}}}=\frac{1}{2}\left(e_{-, \alpha_{\theta}}-e_{+, \alpha_{\theta}}\right) \\
e_{\infty, \alpha_{\theta}}=e_{-, \alpha_{\theta}}+e_{+, \alpha_{\theta}}
\end{array} .\right.
$$

The conformal model of $h_{\theta}\left(\mathbb{R}^{2}\right)$ is

$$
H_{\alpha_{\theta}}=\left\{X_{\alpha_{\theta}}=x_{1} e_{1}+x_{2} e_{2}+\frac{1}{2}\left(x_{1}^{2}+x_{2}^{2}\right) e_{\infty, \alpha_{\theta}}+e_{0, \frac{1}{\alpha_{\theta}}} ; x_{1}, x_{2} \in \mathbb{R}\right\},
$$

where $\varphi_{\alpha_{\theta}}\left(x_{1} e_{1}+x_{2} e_{2}\right)$ models the point $h_{\theta}\left(x_{1} e_{1}+x_{2} e_{2}\right)$ of the perspective plane. Particularly, for all $x_{2} \in \mathbb{R}, \varphi_{\alpha_{\theta}}\left(1 / \sin \theta e_{1}+x_{2} e_{2}\right)$ encodes the point at infinity $h_{\theta}\left(1 / \sin \theta e_{1}+x_{2} e_{2}\right)$. Moreover, since the homogeneous $2-\mathrm{D}$ vector $v_{\theta}(0)$ is equal to $e_{3}$ then $h_{\theta}(0)$ is the origin of $\mathbb{P}_{2}(\mathbb{R})$. Therefore, similarly to the conformal model of $\mathbb{R}^{2}, \varphi_{\alpha_{\theta}}(0)=e_{0, \frac{1}{\alpha_{\theta}}}$ encodes the origin $h_{\theta}(0)$ of the perspective plane $h_{\theta}\left(\mathbb{R}^{2}\right)$.

For the frontal case $\theta=0$, we set

$$
\begin{aligned}
& e_{\infty, \alpha_{0}}=(-1,-1) \\
& e_{0, \frac{1}{\alpha_{0}}}=\frac{1}{2}(1,-1) .
\end{aligned}
$$

The conformal model of $h_{0}\left(\mathbb{R}^{2}\right)$ is reduced to the conformal model of the Euclidean plane $\mathbb{R}^{2}$.

Remark 4.1. We choose here the value -1 for the value of $\alpha_{0}$ because the cot mapping is bijective from $] 0, \pi / 2\left[\right.$ onto $\mathbb{R}_{+}^{*}$. Thus, the negative scalar -1 ensures that the corresponding $e_{\infty, \alpha_{0}}$ is a good representation for the value $\theta=0$. Indeed, it prevents having the same vector $e_{\infty, \alpha_{0}}$ that represents two different latitude angles (one of them is $\theta=0$ ). 


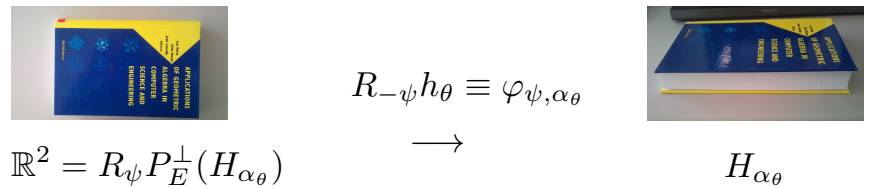

FiguRE 3. Image representation: Image $I_{\psi, \theta}$ whose perspective distortion is encoded by $\varphi_{\alpha_{\theta}}$. Its domain is represented by the horosphere $H_{\alpha_{\theta}}$ (Right). Its associated frontal image $I_{0}$ (Left).

\subsection{Image representation through the conformal model}

In this subsection, the domain of an image previously defined in the projective geometry (equation (44)) is replaced by another one, namely the horosphere, that is a useful geometric object to represent both of the domain and the perspective distortion of the image. This induces a new image representation and a modeling of a single viewpoint change.

Consider the embedding of $\mathbb{R}^{1,1}$ into $\mathbb{R}^{3,1}$ through the conformal split $\mathbb{R}^{3,1}=\mathbb{R}^{2} \oplus \mathbb{R}^{1,1}$, where $\left\{e_{+, \alpha_{\theta}}, e_{-, \alpha_{\theta}}\right\}$ given in the previous section is the orthonormal basis of $\mathbb{R}^{1,1}$. Let $I_{\psi, \theta}$ be an image $I_{\psi, \theta}: R_{-\psi} h_{\theta}\left(\mathbb{R}^{2}\right) \longrightarrow \mathbb{R}$ corresponding to the extrinsic parameters $\left(\theta, \phi, \psi, \lambda, t_{1}, t_{2}\right)$. Let $I_{0}$ be the frontal image associated with $I_{\psi, \theta}$ and corresponding to the extrinsic parameters $\left(0, \phi, 0, \lambda, t_{1}, t_{2}\right)$. Consequently, through the projective pinhole models of acquisition of $I_{\psi, \theta}$ and $I_{0}$, we have:

$$
I_{\psi, \theta} \circ R_{-\psi} h_{\theta}=I_{0}
$$

on the domain of $I_{0}$ encoded by the Euclidean plane $\mathbb{R}^{2}$. As explained previously, the perspective plane $h_{\theta}\left(\mathbb{R}^{2}\right)$ is modeled by $\varphi_{\alpha_{\theta}}\left(\mathbb{R}^{2}\right)$ which is the horosphere $H_{\alpha_{\theta}}$. Next, we need to represent $R_{-\psi}$ in the conformal model associated with $\alpha_{\theta}$ in order to make a full modeling of the domain $R_{-\psi} h_{\theta}\left(\mathbb{R}^{2}\right)$ of $I_{\psi, \theta}$. Let $F_{-\psi}: H_{\alpha_{\theta}} \longrightarrow H_{\alpha_{\theta}}$ defined for $X_{\alpha_{\theta}}=\varphi_{\alpha_{\theta}}(x)$ by

$$
\begin{aligned}
F_{-\psi}\left(X_{\alpha_{\theta}}\right) & =G_{-\psi} X_{\alpha_{\theta}} G_{\psi} \\
& =R_{-\psi}(x)+\frac{1}{2}\left[R_{-\psi}(x)\right]^{2} e_{\infty, \alpha_{\theta}}+e_{0, \frac{1}{\alpha_{\theta}}}
\end{aligned}
$$

where $G_{-\psi}=e^{\frac{\psi}{2} e_{1} \wedge e_{2}}$ is the versor encoding $R_{-\psi}$ in the conformal model associated with $\alpha_{\theta}$ (see equation (25)). Hence, $R_{-\psi} h_{\theta}\left(\mathbb{R}^{2}\right)$ is encoded by $F_{-\psi} \circ \varphi_{\alpha_{\theta}}\left(\mathbb{R}^{2}\right)$ where $F_{-\psi} \circ \varphi_{\alpha_{\theta}}$ is equal to

$$
\varphi_{\alpha_{\theta}} \circ R_{-\psi}=: \varphi_{\psi, \alpha_{\theta}} .
$$

Since $\varphi_{\psi, \alpha_{\theta}}\left(\mathbb{R}^{2}\right)=H_{\alpha_{\theta}}$ for all $\psi$, we encode the domain of $I_{\psi, \theta}$ by $\left\{H_{\alpha_{\theta}}, \varphi_{\psi, \alpha_{\theta}}\right\}$. In fact, the embedding of the domain $\mathbb{R}^{2}$ of $I_{0}$ onto $H_{\alpha_{\theta}}$ is given by $\varphi_{\psi, \alpha_{\theta}}$ that takes into account the parameter $\psi$ of the camera. This leads us to construct the following image representation.

Definition 4.1 (Image conformal representation). Under the above assumptions, an image is given by a conformal embedding $\varphi_{\psi, \alpha_{\theta}}$ of $\mathbb{R}^{2}$ into $\mathbb{R}^{3,1}$ 
corresponding to an orthonormal basis $\left\{e_{+, \alpha_{\theta}}, e_{-, \alpha_{\theta}}\right\}$ of $\mathbb{R}^{1,1}$ that encodes its perspective distortion and a camera rotation parameter $\psi$. It is a mapping $\bar{I}_{\psi, \alpha_{\theta}}$ defined on the associated horosphere as follows:

$$
\begin{aligned}
\bar{I}_{\psi, \alpha_{\theta}}:\left\{H_{\alpha_{\theta}}, \varphi_{\psi, \alpha_{\theta}}\right\} & \longrightarrow \mathbb{R} \\
X_{\alpha_{\theta}}=\varphi_{\psi, \alpha_{\theta}}(x) & \longmapsto I_{\psi, \theta} \circ R_{-\psi} h_{\theta} \circ R_{\psi} P_{E}^{\perp}\left(X_{\alpha_{\theta}}\right) \\
& =I_{\psi, \theta} \circ R_{-\psi} h_{\theta}(x)
\end{aligned}
$$

Remark 4.2. $\quad$ 1. Since $\theta \mapsto \alpha_{\theta}$ is bijective from $\left[0, \frac{\pi}{2}\left[\right.\right.$ onto $\mathbb{R}_{+}^{*} \cup\{-1\}$ then every $I_{\psi, \theta}$ matches with a unique $\bar{I}_{\psi, \alpha_{\theta}}$ and vice versa.

2. On one hand, a vector $X_{\alpha_{\theta}}=\varphi_{\psi, \alpha_{\theta}}(x)$ represents the point $R_{-\psi} h_{\theta}(x)$ that belongs to the image plane of $I_{\psi, \theta}$. On the other hand, $R_{\psi} P_{E}^{\perp}\left(X_{\alpha_{\theta}}\right)$ is the point $x$ in the image plane of $I_{0}$ that matches with $X_{\alpha_{\theta}}$ because it matches with $R_{-\psi} h_{\theta}(x)$ (see figure 3 ).

3. Most of the previous equations are satisfied on subsets of $\mathbb{R}^{2}$ or $H_{\psi, \alpha_{\theta}}$. However, in order to simplify the notations we suppose that we deal with the entire spaces by assuming that the intensity function $I_{\psi, \theta}$ is well defined on the whole of $R_{-\psi} h_{\theta}\left(\mathbb{R}^{2}\right) \subset P_{2}(\mathbb{R})$.

Next, let $\overline{\mathbb{I}}$ denote the set of conformal image representations:

$\overline{\mathbb{I}}=\left\{\bar{I}_{\psi, \alpha_{\theta}}\right.$ s.t $\alpha_{\theta} \in \mathbb{R}_{+}^{*} \cup\{-1\}, \psi \in\left[0,2 \pi\left[, I_{\psi, \theta}: R_{-\psi} h_{\theta}\left(\mathbb{R}^{2}\right) \rightarrow \mathbb{R}\right\}\right.$.

\subsection{Viewpoint change representation}

This subsection is devoted to constructing within the conformal geometry a viewpoint change representation, that is a representation of an homography $k \in \Gamma$.

Following the previous notations, let $\bar{I}_{\psi_{1}, \alpha_{\theta_{1}}}$ and $\bar{I}_{\psi_{2}, \alpha_{\theta_{2}}}$ be two images in $\overline{\mathbb{I}}$ of a planar object given by:

$$
\bar{I}_{\psi_{1}, \alpha_{\theta_{1}}}\left(R_{-\psi_{1}} x+\frac{1}{2}\left(R_{-\psi_{1}} x\right)^{2} e_{\infty, \alpha_{\theta_{1}}}+e_{0, \frac{1}{\alpha_{\theta_{1}}}}\right)=I_{\psi_{1}, \theta_{1}} \circ R_{-\psi_{1}} h_{\theta_{1}}(x)
$$

and

$$
\bar{I}_{\psi_{2}, \alpha_{\theta_{2}}}\left(R_{-\psi_{2}} y+\frac{1}{2}\left(R_{-\psi_{2}} y\right)^{2} e_{\infty, \alpha_{\theta_{2}}}+e_{0, \frac{1}{\alpha_{\theta_{2}}}}\right)=I_{\psi_{2}, \theta_{2}} \circ R_{-\psi_{2}} h_{\theta_{2}}(y) .
$$

We denote by $I_{0}^{1}$ and $I_{0}^{2}$ resp. their associated frontal images (see above). Since $I_{0}^{1}$ and $I_{0}^{2}$ are frontal images of the same planar object, there exists a planar direct similarity $f$ such that

$$
I_{0}^{1}=I_{0}^{2} \circ f .
$$

The homography $k \in \Gamma$ that relates $I_{\psi_{1}, \theta_{1}}$ and $I_{\psi_{2}, \theta_{2}}$ is the mapping given by equation (46):

$$
k: R_{-\psi_{1}} h_{\theta_{1}}(x) \longmapsto x \longmapsto f(x) \longmapsto R_{-\psi_{2}} h_{\theta_{2}}[f(x)] .
$$

According to the previously introduced image representation, it is given by the bijection (see figure 4 ):

$$
\varphi_{\psi_{2}, \alpha_{\theta_{2}}} \circ f \circ \varphi_{\psi_{1}, \alpha_{\theta_{1}}}^{-1}:\left\{H_{\alpha_{\theta_{1}}}, \varphi_{\psi_{1}, \alpha_{\theta_{1}}}\right\} \longrightarrow\left\{H_{\alpha_{\theta_{2}}}, \varphi_{\psi_{2}, \alpha_{\theta_{2}}}\right\} .
$$




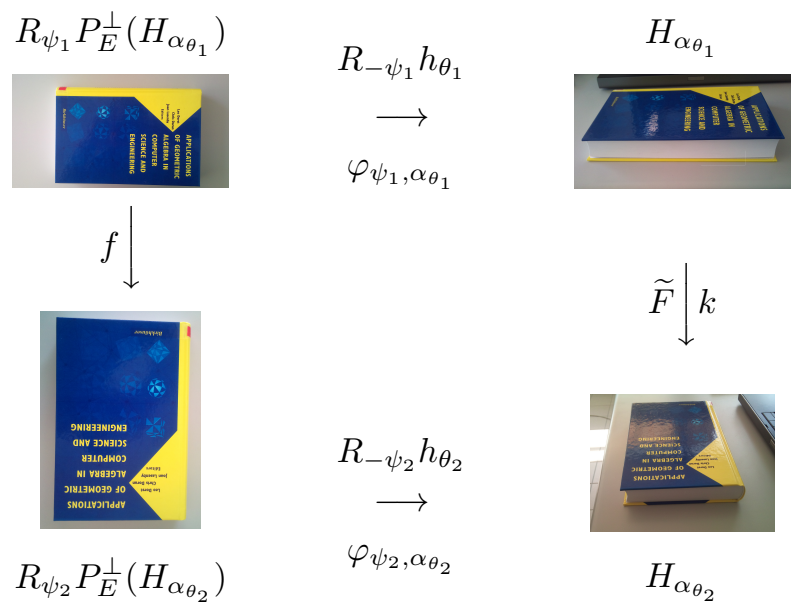

FiguRE 4. Viewpoint change representation: The top row shows the image $I_{\psi_{1}, \theta_{1}}$ (upper right) and its frontal $I_{0}^{1}$ (upper left), the bottom row shows the image $I_{\psi_{2}, \theta_{2}}$ (bottom right) and its frontal $I_{0}^{2}$ (bottom left). The projective mappings and their conformal representations are also shown.

More explicitly, we have the following proposition.

Proposition 4.1. The viewpoint change between the images $\bar{I}_{\psi_{1}, \alpha_{\theta_{1}}}$ and $\bar{I}_{\psi_{2}, \alpha_{\theta_{2}}}$ is represented by the mapping $\widetilde{F}:\left\{H_{\alpha_{\theta_{1}}}, \varphi_{\psi_{1}, \alpha_{\theta_{1}}}\right\} \longrightarrow\left\{H_{\alpha_{\theta_{2}}}, \varphi_{\psi_{2}, \alpha_{\theta_{2}}}\right\}$ that sends

$$
X_{\alpha_{\theta_{1}}}=R_{-\psi_{1}} x+\frac{1}{2}\left(R_{-\psi_{1}} x\right)^{2} e_{\infty, \alpha_{\theta_{1}}}+e_{0, \frac{1}{\alpha_{\theta_{1}}}}
$$

to

$$
\widetilde{F}\left(X_{\alpha_{\theta_{1}}}\right)=R_{-\psi_{2}} f(x)+\frac{1}{2}\left[R_{-\psi_{2}} f(x)\right]^{2} e_{\infty, \alpha_{\theta_{2}}}+e_{0, \frac{1}{\alpha_{\theta_{2}}}} .
$$

This conformal viewpoint change representation is represented by the following diagram:

$$
\begin{array}{ccc}
H_{\alpha_{\theta_{1}}} \stackrel{\widetilde{F}}{\longrightarrow} & H_{\alpha_{\theta_{2}}} \\
\left.\varphi_{\psi_{1}, \alpha_{\theta_{1}}} \uparrow\right|_{R_{\psi_{1}} P_{E}^{\perp}} & R_{\psi_{2} P_{E}^{\perp}} \mid \uparrow_{\varphi_{\psi_{2}, \alpha_{\theta_{2}}}}^{\longrightarrow} \\
\mathbb{R}^{2} \stackrel{f}{\longrightarrow} & \mathbb{R}^{2}
\end{array} .
$$

Remark 4.3. The two isotropic bases $\left\{e_{\infty, \alpha_{\theta_{1}}}, e_{0, \frac{1}{\alpha_{\theta_{1}}}}\right\}$ and $\left\{e_{\infty, \alpha_{\theta_{2}}}, e_{0, \frac{1}{\alpha_{\theta_{2}}}}\right\}$ of $\mathbb{R}^{1,1}$ encode the latitude angles $\theta_{1}$ and $\theta_{2}$ of the perspective distortions of the two images. The rotation parameters $\psi_{1}$ and $\psi_{2}$ of the two cameras are encoded by the rotations $R_{-\psi_{1}}$ and $R_{-\psi_{2}}$. Furthermore, the affine similarity $f$ gives an additional representation of the remaining parameters via its 4 
degrees of freedom: it models the variation of the parameters of the associated frontal images $I_{0}^{1}$ and $I_{0}^{2}$. Hence, $\widetilde{F}$ takes into consideration all the degrees of freedom of the homography $k \in \Gamma$. This means that it is a well-chosen viewpoint change representation.

Remark 4.4. Recall that a transformation $F: \mathbb{R}^{3,1} \longrightarrow \mathbb{R}^{3,1}$ of class $C^{1}$ is said to be conformal if and only if for all $X \in \mathbb{R}^{3,1}$ there exists $\lambda(X) \neq 0$ such that

$$
[d F(X) \cdot x] \cdot[d F(X) \cdot y]=\lambda(X) x \cdot y, \forall x, y \in \mathbb{R}^{3,1},
$$

where $d F$ is the differential function of $F$. Particularly, an orthogonal transformation is conformal.

Proposition 4.2. The mapping $\widetilde{F}$ is the restriction to the horosphere $H_{\alpha_{\theta_{1}}}$ of a linear conformal transformation of $\mathbb{R}^{3,1}$.

Proof. Let $F_{1}: H_{\alpha_{\theta_{1}}} \longrightarrow H_{\alpha_{\theta_{1}}}$ and $F_{2}: H_{\alpha_{\theta_{1}}} \longrightarrow H_{\alpha_{\theta_{2}}}$ be two mappings that send

$$
Y_{1}=y+\frac{1}{2} y^{2} e_{\infty, \alpha_{\theta_{1}}}+e_{0, \frac{1}{\alpha_{\theta_{1}}}}
$$

to

$$
F_{1}\left(Y_{1}\right)=g(y)+\frac{1}{2}[g(y)]^{2} e_{\infty, \alpha_{\theta_{1}}}+e_{0, \frac{1}{\alpha_{\theta_{1}}}}
$$

and

$$
F_{2}\left(Y_{1}\right)=y+\frac{1}{2} y^{2} e_{\infty, \alpha_{\theta_{2}}}+e_{0, \frac{1}{\alpha_{\theta_{2}}}},
$$

respectively, where $g=R_{-\psi_{2}} f R_{\psi_{1}}$. We have

$$
\widetilde{F}=F_{2} \circ F_{1} .
$$

Since $g$ is a planar direct affine similarity, it is the composition of a rotation, translation and dilation with ratio $\rho$ not equal 0 . Therefore, from equation (25) there exists a versor $G_{\alpha_{\theta_{1}}}$ in $\mathbb{R}_{3,1}$ such that

$$
F_{1}\left(Y_{1}\right)=\rho G_{\alpha_{\theta_{1}}} Y_{1} G_{\alpha_{\theta_{1}}}^{*}{ }^{-1} .
$$

Consequently, $F_{1}$ is the restriction to $H_{\alpha_{\theta_{1}}}$ of a conformal transformation of $\mathbb{R}^{3,1}$. Moreover, $F_{2}$ is the restriction to $H_{\alpha_{\theta_{1}}}$ of the conformal transformation of $\mathbb{R}^{3,1}$ that sends a vector

$$
y+y_{\infty} e_{\infty, \alpha_{\theta_{1}}}+y_{0} e_{0, \frac{1}{\alpha_{\theta_{1}}}}
$$

to the vector

$$
y+y_{\infty} e_{\infty, \alpha_{\theta_{2}}}+y_{0} e_{0, \frac{1}{\alpha_{\theta_{2}}}} .
$$

In fact, one can easily verify that this last transform is orthogonal since $e_{\infty, \alpha_{\theta_{1}}} \cdot e_{0, \frac{1}{\alpha_{\theta_{1}}}}=e_{\infty, \alpha_{\theta_{2}}} \cdot e_{0, \frac{1}{\alpha_{\theta_{2}}}}=-1$.

Hence, $\widetilde{F}$ is the restriction to $H_{\alpha_{\theta_{1}}}$ of a conformal transformation of $\mathbb{R}^{3,1}$ 
This viewpoint change representation leads in the next subsection to interpret the pinhole camera model in the conformal geometry instead of the projective geometry as above.

\subsection{Conformal pinhole camera model}

Here, the homography $k_{0}$ defining the acquisition of $I_{\psi, \theta}$ in the projective geometry (equation (43)) and relating the object reflectance $I^{0}$ to $I_{\psi, \theta}$ is modeled by a mapping in the conformal geometry between their associated horospheres.

We remind that $e_{\infty, \alpha_{0}}=(-1,-1)$ and $e_{0, \frac{1}{\alpha_{0}}}=\frac{1}{2}(1,-1)$ are the two isotropic vectors representing the frontal view of the object and $H_{\alpha_{0}}$ the corresponding horosphere. Similarly, if $e_{\infty, \alpha_{\theta}}=\left(\alpha_{\theta}, \alpha_{\theta}\right)$ and $e_{0, \frac{1}{\alpha_{\theta}}}=\frac{1}{2}\left(-\frac{1}{\alpha_{\theta}}, \frac{1}{\alpha_{\theta}}\right)$ are the vectors encoding the latitude angle of $I_{\psi, \theta}$, we denote by $H_{\alpha_{\theta}}$ their associated horosphere. Thus, applying the previous conformal viewpoint change representation, the homography $k_{0}=R_{-\psi} h_{\theta} R_{-\phi} D_{\lambda^{-1}} T$ is represented by the following bijection $F_{0}:\left\{H_{\alpha_{0}}, \varphi_{0, \alpha_{0}}\right\} \rightarrow\left\{H_{\alpha_{\theta}}, \varphi_{\psi, \alpha_{\theta}}\right\}$ that sends

$$
X_{0}=x+\frac{1}{2} x^{2} e_{\infty, \alpha_{0}}+e_{0, \frac{1}{\alpha_{0}}}
$$

to

$$
F_{0}\left(X_{0}\right)=R_{-\psi} f_{0}(x)+\frac{1}{2}\left[R_{-\psi} f_{0}(x)\right]^{2} e_{\infty, \alpha_{\theta}}+e_{0, \frac{1}{\alpha_{\theta}}},
$$

where $f_{0}=R_{-\phi} D_{\lambda^{-1}} T$. This leads to the following definition:

Definition 4.2. Under all these previous notations, the conformal pinhole camera model $\bar{I}_{\psi, \alpha_{\theta}}$ of $I_{\psi, \theta}$ is defined by:

$$
\begin{aligned}
\bar{I}_{\psi, \alpha_{\theta}}:\left\{H_{\alpha_{\theta}}, \varphi_{\psi, \alpha_{\theta}}\right\} & \longrightarrow \mathbb{R} \\
Y & \longmapsto \bar{I}_{0, \alpha_{0}} \circ F_{0}^{-1}(Y)=I^{0} \circ P_{E}^{\perp} \circ F_{0}^{-1}(Y),
\end{aligned}
$$

where

$$
\begin{aligned}
\bar{I}_{0, \alpha_{0}}:\left\{H_{\alpha_{0}}, \varphi_{0, \alpha_{0}}\right\} & \longrightarrow \mathbb{R} \\
X_{0}=x+\frac{1}{2} x^{2} e_{\infty, \alpha_{0}}+e_{0, \frac{1}{\alpha_{0}}} & \longmapsto \bar{I}_{0, \alpha_{0}}\left(X_{0}\right)=I^{0} \circ P_{E}^{\perp}\left(X_{0}\right)=I^{0}(x) .
\end{aligned}
$$

is the conformal representation of $I^{0}$.

\section{Conclusion}

We have shown in this paper how to take advantage of the conformal model to introduce new image and viewpoint change representations. We have for instance described how to deal with the perspective distortion parameter using the two isotropic vectors $e_{\infty, \alpha_{\theta}}$ and $e_{0, \frac{1}{\alpha_{\theta}}}$. This is expressed by the mean of a generalization of the conformal model that represents a perspective plane $h_{\theta}\left(\mathbb{R}^{2}\right)$ for every scalar $\alpha_{\theta}$. This allows to consider an image as defined on a horosphere $H_{\alpha_{\theta}}$ with a given embedding $\varphi_{\psi, \alpha_{\theta}}$ of $\mathbb{R}^{2}$ into $\mathbb{R}_{3,1}$ corresponding to an isotropic basis $\left\{e_{\infty, \alpha_{\theta}}, e_{0, \frac{1}{\alpha_{\theta}}}\right\}$ that encodes the latitude angle $\theta$ and a rotation parameter $\psi$. In this context, every viewpoint change is described 
as a mapping between two horospheres linked by an affine similarity that relates there associated frontal images. In the end, we have modeled the pinhole model of acquisition of an image $I_{\psi, \theta}$ within this setting in the conformal geometry by making use of the conformal viewpoint change representation between $I_{\psi, \theta}$ and the image $I^{0}$ encoding the reflectance of the planar object.

We will detail in a forthcoming paper how to make a group-representation of all the viewpoint changes that acts on the set of images and decomposes $\overline{\mathbb{I}}$ into disjoint union of orbits in the conformal geometry. This will enable us to define viewpoint change invariant detectors and descriptors in this framework using the theory of group actions.

\section{References}

[1] F. Gourd, J-P. Gauthier, and H. Younes. Une méthode d'invariants de l'analyse harmonique en reconnaissance de formes. Traitement du Signal [Trait. Signal], 6:161-178, 1989.

[2] R. I. Hartley and A. Zisserman. Multiple View Geometry in Computer Vision. Cambridge University Press, ISBN: 0521540518, second edition, 2004.

[3] D. Hestenes, H. Li, and A. Rockwood. New algebraic tools for classical geometry. In G. Sommer, editor, Geometric computing with Clifford algebras, pages 3-26. Springer-Verlag, 2001.

[4] D. Hestenes and G. Sobcyk. Clifford Algebra to Geometric Calculus: A Unified Language for Mathematics and Physics. Kluwer Academic Publishers, 1987.

[5] D. Hestenes and R. Ziegler. Projective geometry with clifford algebra. Acta Applicandae Mathematicae, 23:25-63, 1991.

[6] H. Li, D. Hestenes, and A. Rockwood. Generalized homogeneous coordinates for computational geometry. In G. Sommer, editor, Geometric computing with Clifford algebra, pages 27-59. Springer-Verlag, 2001.

[7] D. G. Lowe. Distinctive image features from scale-invariant keypoints. International Journal of Computer Vision, 2(60):91-110, 2004.

[8] J. L. Mundy and A. Zisserman. Appendix - projective geometry for machine vision. In J. L. Mundy and A. Zisserman, editors, Geometric invariance in computer vision, pages 463-519. MIT Press, Cambridge, MA, USA, 1992.

[9] C. Perwass. Applications of geometric algebra in computer vision. PhD thesis, University of Cambridge, 2000.

[10] C. Perwass and D. Hildenbrand. Aspects of geometric algebra in euclidean, projective and conformal space. Technical Report 0310, Christian-Albrechts University, 2003.

[11] S. Soatto. Steps towards a theory of visual information: Active perception, signal-to-symbol conversion and the interplay between sensing and control. arxiv:1110.2053, October 2011.

[12] G. Yu and J. M. Morel. ASIFT: An algorithm for fully affine invariant comparison. Image Processing On Line, 2011. 
Ghina El Mir

MIA Laboratory, La Rochelle University, La Rochelle, France e-mail: ghina.elmir@univ-lr.fr

Christophe Saint-Jean

MIA Laboratory, La Rochelle University, La Rochelle, France e-mail: christophe.saint-jean@univ-lr.fr

Michel Berthier

MIA Laboratory, La Rochelle University, La Rochelle, France e-mail: michel. berthier@univ-lr.fr 